\title{
Equinodermos de las Islas Lobos de Afuera (Lambayeque, Perú)
} Echinoderms from Lobos de Afuera islands (Lambayeque, Peru)

\author{
Yuri Hooker ${ }^{1}$, Francisco A. Solís-Marín ${ }^{2}$, Miguel Lleellish ${ }^{3}$ \\ Presentado: 06/07/2005 \\ Aceptado: $\quad 09 / 08 / 2005$
}

\section{Resumen}

Del 19 al 24 de junio de 1999 se llevó a cabo una expedición para realizar el inventario rápido de los equinodermos de las Islas Lobos de Afuera $\left(06^{\circ} 55,5 \mathrm{~S}-80^{\circ} 42,5 \mathrm{~W}\right)$. Se colectó un total de 39 especies de equinodermos: 7 asteroideos (2 nuevos registros para el Perú: Luidia bellonae, Luidia brevispina); 8 ofiuroideos (1 nuevo registro para el Perú: Ophionereis sp.); 11 equinoideos (5 nuevos registros para el Perú: Astropyga pulvinata, Diadema mexicanum, Lytechinus sp., Toxopneustes roseus, Tripneustes depressus) y 13 holoturoideos (6 nuevos registros: Psolidium dorsipes, Cucumaria flamma, Pseudocnus californicus, Holothuria sp., Chiridota sp. y Actinopyga sp.).

Palabras Clave: Echinodermata, nuevos registros, islas Lobos de Afuera, biodiversidad, Perú.

\section{Abstract}

A rapid survey in Islas Lobos de Afuera $\left(06^{\circ} 55,5 \mathrm{~S}-80^{\circ} 42,5 \mathrm{~W}\right)$ were carry out from June 19 to 24 of 1999. A total of 39 Echinodermata species was collected: 7 Asteroidea, including two new records for Peru, Luidia bellonae, Luidia brevispina; 8 Ophiuroidea, whit one new record for Peru Ophionereis sp.; 11 Echinoidea, including five new records for Peru, Astropyga pulvinata, Diadema mexicanum, Lytechinus sp., Toxopneustes roseus, Tripneustes depressus; and 13 Holoturoidea, including six new records, Psolidium dorsipes, Cucumaria flamma, Pseudocnus californicus, Holothuria sp., Chiridota sp. and Actinopyga sp.

Keywords: Echinodermata, Lobos de Afuera islands, new records, Biodiversity, Peru.

\section{Introducción}

Uno de los grupos taxonómicos de mayor importancia en la estructura de las comunidades marinas, es el Phylum Echinodermata (los equinodermos). Su presencia es conspicua en todos los ambientes marinos, desde la zona intermareal hasta los abismos oceánicos y desde las fuentes hidrotermales submarinas hasta las aguas polares. Su éxito en la colonización del mar se debe a las adaptaciones que han venido desarrollando desde su aparición antes del Cámbrico Inferior (más de 600 millones de años). En los ecosistemas marinos se encuentran equinodermos en diferentes ni-

(1)Laboratorio de Biología Marina, Facultad de Ciencias y Filosofia, Universidad Peruana Cayetano Heredia. Calle Honorio Delgado 430, Lima 31, Lima, Perú.

(2)Laboratorio de Sistemática y Ecología de Equinodermos, Colección Nacional de

Equinodermos, Universidad Autónoma de México.

(3)Dirección de Conservación de la Biodiversidad, Instituto Nacional de Recursos Naturales.

E-mail Yuri Hoocker: hookery@yahoo.com veles tróficos: herbívoros, sedimentívoros, carroñeros, carnívoros y omnívoros.

En el Pacífico Oriental los equinodermos son uno de los grupos mejor conocidos (Holgín et al, 2000), no obstante los estudios taxonómicos de los equinodermos peruanos son escasos, siendo la publicación más reciente la de Clark (1910), donde hace un recuento y descripción de las especies colectadas por él.

Caso $(1978,1979)$ y Maluf (1988), mencionan a Perú como límite sur de la distribución de un importante número de especies tropicales del Pacífico Oriental, y límite norte de especies de la Corriente Peruana. Lo anterior principalmente basado en registros realizados por expediciones del siglo 19 (Challenger 1873-76 y los trabajos de E. Verrill entre 1867 y 1914).

La localidad donde se desarrolló el presente estudio es de singular interés científico. Las islas Lobos de Afuera (0655,5 S - 8042,5 W) constituyen el límite austral de la distribución de muchas especies de la Provincia Bioecológica Panámica. Esto, debido a que su distancia a la 
costa (33 millas) permite que la Corriente Costera Peruana tenga poca influencia sobre la fauna de estas islas, manteniendo sus características básicamente tropicales. Las islas muestran gran variedad de hábitats submarinos como acantilados, arrecifes rocosos, rocas de derrumbe, cantos rodados, fondos arenosos, fondos de cascajo, entre otros, factor preponderante para la existencia de una gran biodiversidad.

El presente trabajo proporciona una lista de especies de equinodermos, así como los hábitats en que fueron encontrados en la localidad de islas Lobos de Afuera.

\section{Material y métodos}

Las colectas se desarrollaron entre el $19 \mathrm{y}$ el 24 de junio de 1999, por medio de buceo con compresora (hooka) en recorridos desde la zona intermareal hasta los $20 \mathrm{~m}$ de profundidad, mediante tres faenas diarias de buceo de aproximadamente 3 horas cada una (matutinos, vespertinos y nocturnos).

Por las condiciones adversas del mar (alta dinámica y/o presencia de marea roja), solo se recolectó en 8 estaciones situadas en zonas protegidas del oleaje (Fig. 1). El área aproximada de muestreo por estación fue de 1 hectárea cuadrada. La mayoría de estaciones presentaron una gran diversidad de hábitats, siendo las especies recolectadas indicadoras de las mismas.

En cada operación de buceo y en cada tipo de hábitat, se registraron datos de ocurrencia de especies, número aproximado de especimenes observados y profundidad de registro, con lo cual se elaboró una escala cualitativa de abundancia: $\mathrm{R}$ (Rara) $=1$ o 2 especímenes; F (Frecuente) $=3$ a 10 especímenes; $\mathrm{C}$ (Común $)=11$ a 50 especímenes; A (Abundante) $=$ más de 50 especímenes (Fig. 2).

Las colectas se realizaron manualmente, utilizándose en ocasiones, cuchillos para remover rocas. Se usó una bolsa tamiz de 0,5 mm para cernir substratos blandos y detectar equinodermos enterrados.

Los especímenes recolectados fueron llevados a la estación en tierra donde fueron adormecidos usando sulfato de sodio (aproximadamente 50 g por litro) para manipularlos y fijados en alcohol etílico al 70\%. Terminadas las colectas, en el laboratorio se procedió a secar las muestras en estufa, a $40{ }^{\circ} \mathrm{C}$, por tres días. El proceso se completó a temperatura ambiente por un mes, luego se conservaron secos en envases plásticos herméticos. Los holoturoideos se mantuvieron conservados en frascos plásticos con alcohol al 70\%.

Figura 1. Mapa de ubicación de la Isla Lobos de Afuera. Las estrellas muestran los lugares de estudio

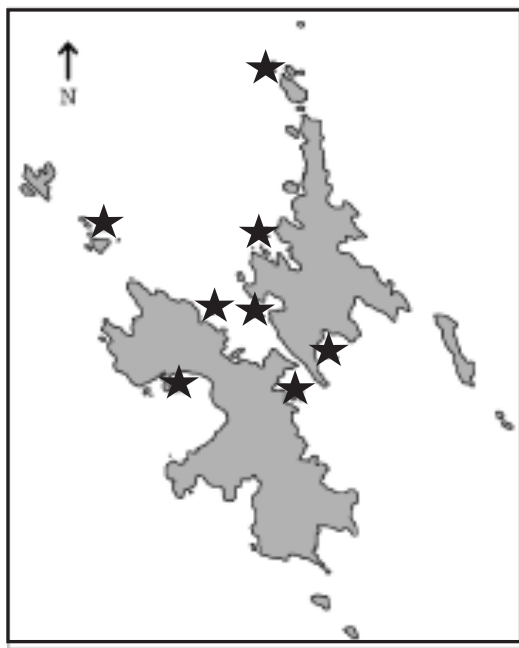

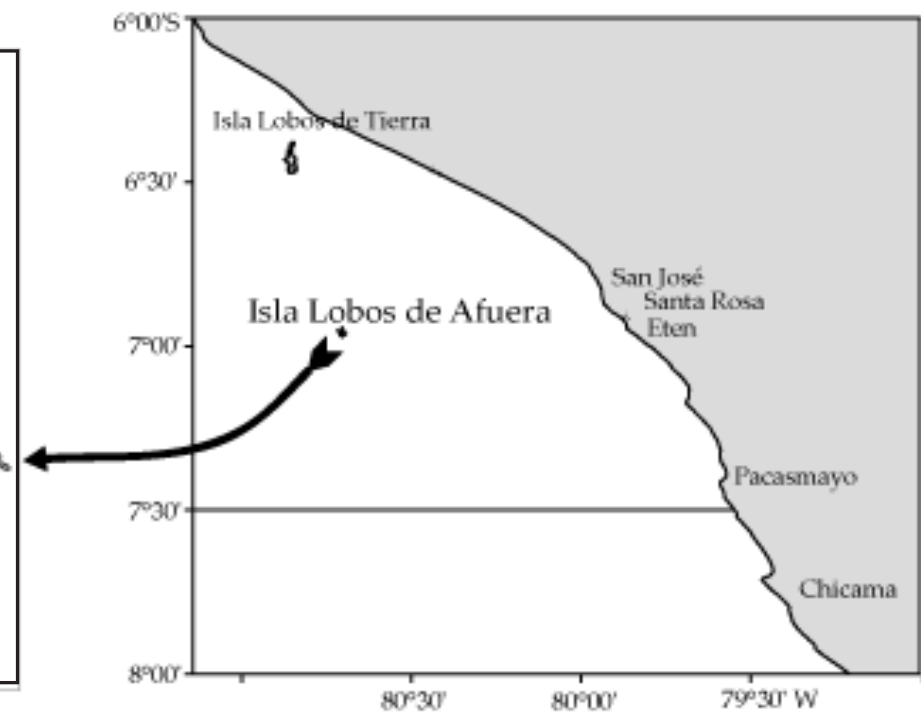

Rev. peru. biol. 12(1): 77-82 (2005) 
Parte de las muestras conservadas fueron identificadas en las instalaciones del albergue de las islas, mientras otras fueron transportadas para su posterior identificación en los laboratorios del IMARPE utilizando los criterios de Clark (1910), Caso (1978, 1979), Castillo(1968), Cleveland y Hickman (1998) y Solís-Marín y Mata (1999). La distribución geográfica considerada fue la propuesta por Maluf (1988).

Las muestras han quedado depositadas en la colección científica del Instituto del Mar del Perú (sin número de código). Elúnico especimen de Actinopyga sp. y otro de Holothuria sp. están depositados en la colección del Laboratorio de Biología Marina de la Universidad Peruana Cayetano Heredia (LBMUPCH-32; LBMUPCH-33, respectivamente).

\section{Resultados}

La comunidad de equinodermos encontrada fue típicamente tropical con un amplio predominio de especies de la Provincia Biogeográfica Panámica, con la presencia de dos especies de amplio rango de distribución, tanto en aguas tropicales como templadas (Heliaster helianthus y Caenocentrotus gibbosus) y una sola especie de la Provincia Peruana (Arbacia spatuligera).

Se colectó un total de 39 especies de equinodermos: 7 asteroideos; 8 ofiuroideos; 11 equinoideos y 13 holoturoideos (Tabla 1). Se incluyen a Luidia brevispina, Pentaceraster cumingi, Actinopyga sp. y Holothuria sp. registradas posteriormente (marzo del 2000), durante una expedición para inventariar los peces de estas islas.

Del total de especies, 15 son nuevos registros para aguas peruanas: Luidia bellonae, Luidia brevistina, Astropyga pulvinata, Diadema mexicanus, Toxopneustes roseus, Tripneustes depressus, Psolidium dorsipes, Chiridota sp. Cucumaria flamma, Pseudocnus californicus, Ophionereis sp., Lytechinus sp., Actinopyga sp., Holothuria sp. Las ultimas 4 especies aparentemente serán nuevas para la ciencia.

\section{Uso de hábitat}

\section{Intermareal rocoso}

El intermareal fue uno de los niveles con menos presencia de equinodermos. Sólo dos especies fueron encontradas: Heliaster helianthus colectándose 2 especímenes, y Arbacia incisa, de la cual se colectó, en una poza intermareal, un raro ejemplar que tenía las columnas ambulacrales dorsales intensamente rojas, siendo la coloración normal, negra.

Es posible que existan otras especies de equinodermos, especialmente ofiuroideos, entre las colonias de percebes (Pollicipes elegans) y mitílidos de las áreas expuestas de mayor rompiente, pero no fue posible colectarlas debido a la alta dinámica del mar durante los días de muestreo.

\section{Fondos arenosos}

Como especies típicas de fondos arenosos, sin presencia de rocas, solamente se encontró un espécimen de Luidia brevispina y un especimen de Ophionereis sp., este último fue recolectado durante buceo nocturno. Luidia bellonae, fue frecuente en este hábitat, siendo una de las especies más comunes en Lobos de Afuera. Esta especie también fue frecuente sobre rocas cubiertas de arena.

En la arena atrapada entre rocas de derrumbe se recolectaron 2 grandes $(30 \mathrm{~cm})$ y raros especímenes del género Holothuria sp., que no concuerdan con las especies descritas para el área y no han podido ser identificados, siendo muy probablemente ejemplares de una nueva especie para la ciencia. Un ejemplar de Actinopyga sp., también fue encontrado en este hábitat, siendo el primer registro de este género en el Pacífico Oriental.

En el sedimento arenoso con partículas de conchuela depositada debajo de rocas de derrumbe, se encontraron enterradas Holothuria impatiens, Holothuria arenicola y un espécimen de Chiridota sp.

\section{Rocas de derrumbe}

Sobre la superficie de rocas angulares caídas al mar desde cerros y acantilados terrestres, se en- 
contró 2 especímenes de Pentaceraster cumingi, y uno de Pharia piramidatus, así como numerosos ejemplares del erizo Caenocentrotus gibbosus.

Debajo de las rocas, en el estrecho espacio entre estas y el substrato, se hallaron a Ophioderma panamense, Ophiocoma aethiops, Ophiocoma sp., Holothuria kefersteini y Pseudocnus californicus, mientras que, en los espacios entre las rocas, se encontraron varios especímenes de Isostichopus fuscus.

Sobre rocas de mayor tamaño (más de 1 $\mathrm{m})$ en aguas más profundas se hallaron a Nidorelia armata, Pharia piramidatus, Lytechinus semituberculatus, Lytechinus sp., Toxopneustes roseus, Tripneustes depressus, Caenocentrotus gibbosus e Isostichopus fuscus.

\section{Orilla rocosa submareales}

Inmediatamente debajo de la zona intermareal, sobre plataformas y terrazas rocosas con aguas agitadas, se hallaron densas colonias mixtas de las pequeñas holoturias Neothyone gibber y Neothyone gibbosa, así como algunos especimenes de Holothuria theeli. La holoturia Cucumaria flamma también fue frecuente.

\section{Paredes rocosas verticales}

Sobre las paredes verticales de acantilados submarinos y arrecifes rocosos, en aguas poco profundas, fueron numerosos los especímenes de Arbacia incisa mientras que, en aguas más profundas, Arbacia spatuligera y Caenocentrotus gibbosus fueron las especies dominantes. También fue frecuente Tripneustes depressus.

\section{Arrecifes rocosos}

Sobre arrecifes sumergidos de bahías con aguas muy tranquilas se hallaron agregaciones numerosas de Astropyga pulvinata y Diadema mexicanum. También compartió este hábitat Centrostephanus coronatus, pero se les encontró solos o en parejas.

En las cúspides de arrecifes emergentes o muy superficiales y con aguas turbulentas, se encontraron colonias compactas de la pequeña holoturia Psolidium dorsipes, especie adaptada a hábitats con aguas agitadas y muy oxigenadas.

\section{Grietas entre las rocas}

Una especie típica de grietas estrechas fue Ophiothrix spiculata, la que oculta su cuerpo en la parte profunda de la grieta siendo solo

Figura 2. Distribución batimétrica de los equinodermos de las islas Lobos de Afuera. Donde: $R$ (Rara); F (Frecuente); C (Común); A (Abundante). Líneas gruesas indican rango de profundidad con mayor abundancia.

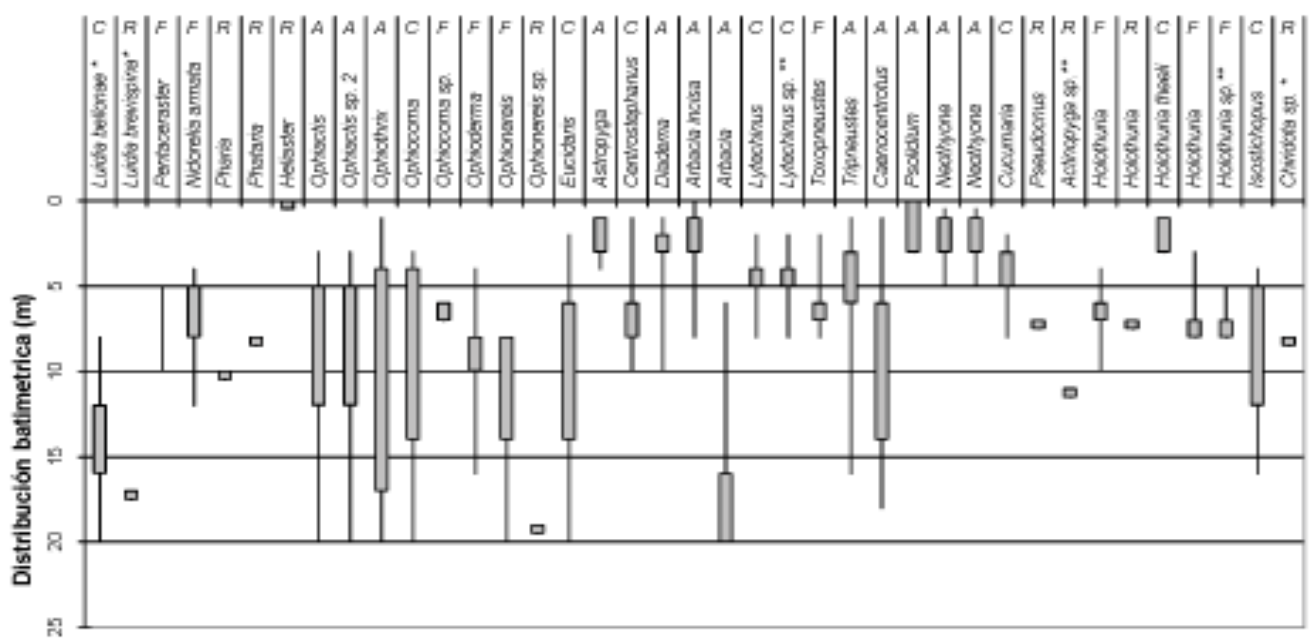


evidentes los brazos que están expuestos por fuera, con los cuales capturan alimento. Otra especie frecuente en grietas fue el erizo Eucidaris thouarsii, quien habitualmente es encontrado anclado a las paredes de las grietas por medio de sus fuertes espinas. Según observaciones realizadas en Máncora y en estas islas, E. thouarsii es depredado por peces como Balistes polylepis y Semicossyphus Darwin.

\section{Algas y gorgonias}

En este hábitat se encontraron dos especies de ofiuroideos de preferencias epibiontes como Ophiactis mirabilis y Ophiactis sp.2, cuyas identificaciones aún están por confirmar. Estas especies están adaptadas para per- manecer enredadas entre las delgadas frondas y ramas de algas y gorgonias, permaneciendo mimetizadas. Sin embargo su número fue muy escaso, a diferencia del encontrado en las playas de la costa norte del Perú.

\section{Distribución batimétrica}

A pesar de que los trabajos se realizaron sólo hasta los $20 \mathrm{~m}$ de profundidad, se observa la zonación de las especies, que tienen una marcada preferencia por determinados estratos batimétricos. Sin embargo, el mayor número de especies y la mayor abundancia se da en los primeros 10 metros y solo Arbacia spatuligera y Luidia bellonae son abundantes a más de 15 m (Fig. 2).

Tabla 1. Especies de equinodermos registradas en las islas Lobos de Afuera. Junio de 1999.

\begin{tabular}{|c|c|c|}
\hline & ASTEROIDEA & ECHINOIDEA \\
\hline & Luidiidae & Arbaciidae \\
\hline 1 & Luidia bellonae Clark, 1917 * & 20 Arbacia incisa (Agassiz, 1863) \\
\hline 3 & Luidia brevispina (Gray, 1840) * & 21 Arbacia spatuligera (Valenciennes, 1846) \\
\hline & Oreasteridae & Toxopneustidae \\
\hline 3 & Pentaceraster cumingi (Gray, 1840) & 22 Lytechinus semituberculatus (Agassiz \& Desor, 1846) \\
\hline 4 & Nidorelia armata (Gray, 1840) & 23 Lytechinus sp. ** \\
\hline & Ophidiasteridae & 24 Toxopneustes roseus (Agassiz, 1863) * \\
\hline 5 & Pharia piramidatus (Gray, 1840) & 25 Tripneustes depressus (Agassiz, 1863) * \\
\hline 6 & Phataria unifascialis (Gray, 1840) & Echinometridae \\
\hline & Heliasteridae & 26 Caenocentrotus gibbosus (Agassiz \& Desor, 1846) \\
\hline 7 & Heliaster helianthus (Lamarck, 1816) & HOLOTHUROIDEA \\
\hline & OPHIUROIDEA & Psolidae \\
\hline & Ophiactidae & 27 Psolidium dorsipes Ludwig, 1886 * \\
\hline 8 & Ophiactis mirabilis & Sclerodactylidae \\
\hline 9 & Ophiactis sp. 2 & 28 Neothyone gibber (Selenka, 1867) \\
\hline & Ophiotrichidae & 29 Neothyone gibbosa Deichmann, 1941 \\
\hline 10 & Ophiothrix spiculata Le Conte, 1851 & Cucumariidae \\
\hline & Ophiocomidae & 30 Cucumaria flamma Solis \& Laguarda 1999 * \\
\hline 11 & Ophiocoma aethiops Lutken, 1859 & 31 Pseudocnus californicus (Semper, 1868) * \\
\hline 12 & Ophiocomasp. & Holothuriidae \\
\hline & Ophiodermatidae & 32 Actinopyga sp.** \\
\hline 13 & Ophioderma panamense Lutken, 1859 & 33 Holothuria impatiens (Forskaal, 1775) \\
\hline & Ophionereidae & 34 Holothuria arenicola Semper, 1868 \\
\hline 14 & Ophionereis annulata (Le Conte, 1851) & 35 Holothuria theeli (Deichmann, 1938) \\
\hline 15 & Ophionereis sp. ** & 36 Holothuria kefersteini (Selenka, 1867) \\
\hline & ECHINOIDEA & 37 Holothuria sp.** \\
\hline & Cidaridae & Stichopodidae \\
\hline 16 & Eucidaris thouarsi (Valenciennes, 1846) & 38 Isostichopus fuscus (Ludwig, 1875) \\
\hline & Diadematidae & Chiridotidae* \\
\hline 17 & Astropyga pulvinata (Lamarck, 1816) * & 39 Chiridota sp. * \\
\hline 18 & Centrostephanus coronatus (Verrill, 1867) & \\
\hline 19 & Diadema mexicanum Agassiz, 1863 * & \\
\hline
\end{tabular}

(*) Nuevo registro para el Perú

(**) Posible nueva especie 


\section{Discusión}

Considerando el poco tiempo empleado para el trabajo de muestreo ( 5 días de trabajo efectivos), además de haber sido evaluadas las zonas expuestas al oleaje, se encontró una alta riqueza de especies en las Islas Lobos de Afuera (39 especies).

Si comparamos los resultados con los trabajos realizados en el Pacífico de México, en localidades de amplitud semejante, encontramos que las Islas Lobos de Afuera son más diversas. Caso (1979), hace un recuento de la diversidad de especies en la bahía de Mazatlán, México, con información recolectada en 3 excursiones (1947, 1970 y 1977) de 12 días cada una; en esta bahía fueron registradas 37 especies de Equinodermos: 17 Asteroideos; 7 Ofiuroideos; 7 Equinoideos y 6 holoturoideos. Por otro lado Holguín et al. (2000) evaluó durante 3 meses la riqueza de equinodermos en fondos someros de la Bahía de Loreto, Baja California Sur, México, incluyendo las 3 islas y la línea de costa de la zona, encontrando un total de 26 especies.

La importancia biogeográfica de las islas se demuestra por la presencia de especies que amplían notablemente su distribución, como Chiridota sp. La familia Chiridotidae sólo estaba registrada para el hemisferio norte, entre California y Panamá. Este es el primer registro de la familia en el hemisferio sur.

El registro del género Actinopyga en el Pacífico Oriental, es un hallazgo notable si consideramos que solo era conocido en el Indopacífico. Los grandes especímenes no identificados de Holothuria sp. no corresponden a ninguno de los Holoturoideos conocidos en el Pacífico Oriental. Esta especie también la hemos registrado en la zona de Máncora. Ambas especies son probablemente nuevas para la ciencia.

Es importante continuar las investigaciones sobre equinodermos y otros grupos taxonómicos en el área estudiada porque, además de ser el límite sur de distribución de la fauna Panámica, las Islas Lobos de Afuera podrían ser un área de endemismos, dada la distancia de la costa pudiendo ser el último refugio de especies tropicales en el Pacífico Sur Este.

La información proporcionada en el presente trabajo puede coadyuvar a las estrategias de conservación de esta importante localidad geográfica que, por su alta diversidad, merecería ser considerada como Santuario Nacional por el Sistema Nacional de Áreas Naturales Protegidas por el Estado (SINANPE).

\section{Agradecimientos}

La presente investigación fue posible gracias al financiamiento otorgado por el Instituto del Mar del Perú. Deseamos agraceder en forma especial a Albetina Kameya por hacer posible esta expedición y el apoyo y hospitalidad de la Dirección de Hidrografía y Navegación de la Marina de Guerra del Perú brindada en sus instalaciones en las islas Lobos de Afuera.

\section{Literatura citada}

Caso, M.E. 1978. Los equinodermos del Pacífico de México. Parte primera: Ordenes Cidaroidea y Aulodonta. Parte segunda - Ordenes Stiridonta y Camarodonta. Centro Cienc. del Mar y Limnol. UNAM, Publ. Esp. 1:1-244

Caso, M.E. 1979. Los Equinodermos de la Bahía de Mazatlán, Sinaloa. Centro Cienc. del Mar y Limnol. Univ. Nal. Autón. México. 6(1):167-368

Castillo, J. 1968. Contribución al Conocimiento de los ophiuroideos Chilenos. Gayana, Instituto Central de Biología. Universidad de Concepción. $\mathrm{N}^{\mathrm{o}} 14,81 \mathrm{pp}$.

Clark, H. 1910. The Echinoderms of Peru. Bulletin of the Museum of Comparative Zoology at Harvard University. 52(17):321-358.

Cleveland P. y J. Hickman, 1998. A Field Guide to Sea Stars \& Other Echinoderms of Galapagos. Sugar Spring Press. 83 pp.

Holguín O.; H. Wright, y F. Solís-Marín, 2000. Asteroidea, Echinoidea y Holothuroidea en fondos someros de la Bahía de Loreto, Baja California Sur, México. Revista de Biología Tropical 48(4): 749-757

Maluf, L. 1988.Composition and Distribution of the Central Eastern Pacific Echinoderms. Natural History Museum of Los Angeles County. Technical Reports, No2. 242 pp.

Solís-Marín, F. y E. Mata. 1999. Manual: Curso Taller Taxonomía de Equinodermos. Universidad Autónoma de México. 88 pp. 